\title{
AGO Recommendations for Diagnosis and Treatment of Patients with Early and Metastatic Breast Cancer: Update 2012
}

\author{
Anton Scharl $^{\mathrm{a}}$ Christoph Thomssen ${ }^{\mathrm{b}}$ Nadia Harbeck $^{\mathrm{c}}$ on behalf of the AGO Breast Committee* \\ ${ }^{a}$ Brustzentrum, Frauenklinik, Klinikum St. Marien, Amberg, \\ ${ }^{b}$ Klinik und Poliklinik für Gynäkologie, Martin-Luther-Universität Halle-Wittenberg, Halle (Saale), \\ 'Brustzentrum der Universität München, Frauenkliniken Großhadern und Maistraße-Innenstadt, Munich, Germany
}

For the last 11 years, the Breast Committee of the Arbeitsgemeinschaft Gynäkologische Onkologie (German Gynecological Oncology Group, AGO) has been preparing and updating evidence-based recommendations for the diagnosis and treatment of patients with early breast cancer (EBC) and metastatic breast cancer (MBC). The AGO Breast Committee consists of 44 gynecological oncologists specialized in breast cancer and interdisciplinary members specialized in pathology, radiological diagnostics, medical oncology, and radiotherapy (RT). The update is performed according to documented rules by thoroughly reviewing and scoring, section by section, the recent publications for their scientific validity (Oxford Level of Evidence (LoE); www.cebm.net [34]) and their clinical relevance (AGO grades of recommendation;

*Members of the 'AGO Breast Committee' in alphabetical order: UteSusan Albert, Marburg; Ingo Bauerfeind, Landshut; Joachim Bischoff, Magdeburg; Jens Uwe Blohmer, Berlin; Klaus Brunnert, Osnabrück; Peter Dall, Lüneburg; Ingo J. Diel, Mannheim; Tanja Fehm, Tübingen; Nikos Fersis, Chemnitz; Michael Friedrich, Krefeld; Kay Friedrichs, Hamburg; Bernd Gerber, Rostock; Volker Hanf, Fürth; Nadia Harbeck, München; Jens Huober, Düsseldorf; Christian Jackisch, Offenbach; Wolfgang Janni, Düsseldorf (Co-Chair); Walter Jonat, Kiel (DKH); Hans H. Kreipe, Hannover (DGP); Sherko Kümmel, Essen; Sibylle Loibl, NeuIsenburg; Hans-Joachim Lück, Hannover; Michael Lux, Erlangen; Nicolai Maass, Aachen; Gunter von Minckwitz, Neu-Isenburg; Volker Möbus, Frankfurt/M.; Christoph Mundhenke, Kiel; Volkmar Müller, Hamburg; Ulrike Nitz, Mönchengladbach; Mahdi Rezai, Düsseldorf; Achim Rody, Frankfurt; Anton Scharl, Amberg (Chair); Rita Schmutzler, Köln; Marcus Schmidt, Mainz; Andreas Schneeweiß, Heidelberg (AIO); Ingrid Schreer, Kiel (DGS); Florian Schütz, Heidelberg; Peter Sinn, Heidelberg (Pathologie); Erich F. Solomayer, Homburg; Rainer Souchon, Tübingen (ARO); Elmar Stickeler, Freiburg i.Br.; Christoph Thomssen, Halle (Saale); Michael Untch, Berlin table 1). Here, we present the 2012 update of these guidelines focusing on modifications performed for the 2012 version. The full version of the update is available online in PDF format (www.ago-online.de, 'Leitlinien') [2] in an English and a German version.

\section{Early Detection and Diagnosis}

Considering early detection and diagnosis of breast cancer, there were no changes or modifications of the current recommendations compared to 2011. New data from 2 recent trials from The Netherlands [193] and Sweden [97], however, confirm the AGO recommendation for mammographic screening also in younger women $40-50$ years of age ( $\mathrm{LoE} 1 \mathrm{bB}, \mathrm{AGO}+)$.

\section{Breast Cancer Risk and Prevention}

There were no clinically relevant changes in the AGO recommendations concerning breast cancer risk and prevention compared to 2011. On the other hand, there were some important publications stimulating further investigations. In 2010, RAD51C was identified as the 3rd high-risk gene for breast and ovarian cancer, which is, however, rarely mutated [124]. RAD51C constitutes a proof of concept for the existence of more, yet very rarely affected high-risk genes. Recently, it was further shown by Loveday et al. [117] that germline mutations in RAD51D may confer susceptibility to ovarian cancer.

Poly(ADP-ribose) polymerase (PARP) inhibitors impair base excision repair during mitosis [26, 67]. In patients with metastatic triple-negative breast cancer (breast cancer antigen (BRCA) deficient), phase II studies with PARP inhibitors

\section{KARGER}

Fax +497614520714

Information@Karger.de

www.karger.com (c) 2012 S. Karger GmbH, Freiburg

$1661-3791 / 12 / 0074-0322 \$ 38.00 / 0$

Accessible online at:

www.karger.com/brc
Prof. Dr. Anton Scharl

Brustzentrum, Frauenklinik

Klinikum St. Marien

92224 Amberg, Germany

Tel. +49 9621 3813-71, Fax -58

scharl.anton@klinikum-amberg.de 
Table 1. AGO grades of recommendation

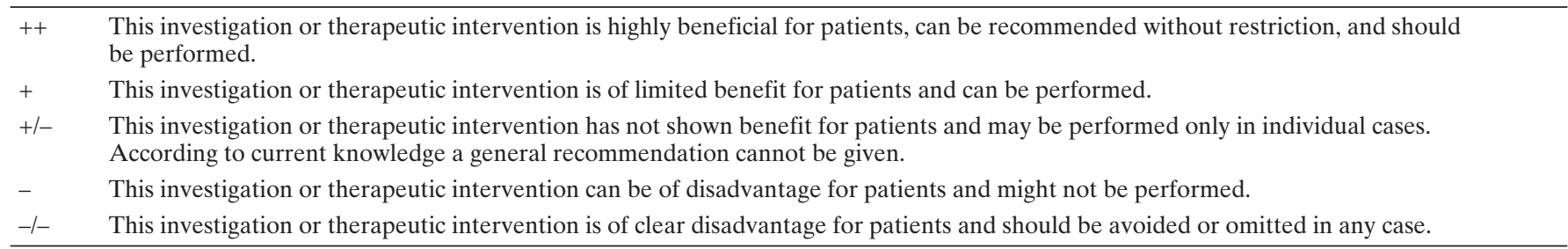

suggested that this genetically defined subgroup might benefit from PARP inhibitor treatment [143]. However, in a phase III trial, efficacy has not been confirmed on a significant level [92].

In a large prospective trial $(\mathrm{n}=4560)$ including postmenopausal women with a moderately increased life-time risk to develop invasive breast cancer, the aromatase inhibitor (AI) exemestane significantly reduced invasive breast cancer occurrence. Whether the lowered incidence also transfers into reduced breast cancer-related mortality is still under investigation [88] (AGO recommendation unchanged: LoE 1bA, $\mathrm{AGO}+)$

\section{Lesions of Uncertain Malignant Potential (B3) - ADH, LIN, FEA}

In the 2012 update, the AGO recommendations concerning lobular intraepithelial neoplasia (LIN) were modified. LIN includes atypical lobular hyperplasia (ALH) and lobular carcinoma in situ (LCIS/CLIS). LIN is categorized as lesion of uncertain malignant potential (B3) if the criteria for pleomorphic LIN and LIN with necrosis or LIN with extensive involvement of lobules are not fulfilled, which may qualify for B5a. These lesions are considered to carry an increased risk $[74,142,180]$, but there is only little evidence to support this conclusion. A grading system (LIN 1 to LIN 3) has been suggested as a tool for a more precise estimation of the individual risk; however, validation of this system has yet to be performed $[168,180]$.

Since LIN is frequently associated with invasive cancer, which may not be represented in core needle or vacuumassisted biopsies, open excisional biopsy is necessary if careful correlation with imaging is inconclusive ( $\mathrm{LoE} 2 \mathrm{bC}, \mathrm{AGO}++$ ) [31, 65, 134, 170, 155].

LIN at the margins of a resection specimen is considered as incidental finding; thus, no further surgery or re-excision has to be performed, provided that the imaging abnormality has been removed ( $\mathrm{LoE} 3 \mathrm{aC}, \mathrm{AGO}++)$ [24, 65, 161]. Likewise, LIN accompanying intraductal or invasive carcinoma in patients with breast-conserving surgery (BCS) necessitates no further resection ( $\mathrm{LoE} 2 \mathrm{aC}, \mathrm{AGO}++$ ). Nevertheless, if pleomorphic LIN or LIN with necrosis is diagnosed, complete resection of the lesion is advised (LoE 5D, AGO ++) [39].

\section{Ductal Carcinoma in situ}

Only little clinically relevant modifications were made in 2012. Focality is considered as a strong prognostic factor with regard to the risk of local-/locoregional recurrence in ductal carcinoma in situ (DCIS). However, biological factors as estrogen receptor (ER), progesterone receptor (PR), human epidermal growth factor receptor 2 (HER2), cyclooxygenase (COX), p16, or Ki-67 do not add relevant prognostic information. Also, the recently developed polymerase chain reaction (PCR)-based multi-gene DCIS score [214] requires further validation before being recommended for routine clinical use.

\section{Pathology}

Pathological diagnosis is crucial and the prerequisite of diagnosis, prognostic assessment, surgical, radiotherapeutical and medical therapy. It includes histopathological diagnosis of needle biopsy specimens, correlation with the imaging [73], differential diagnosis of the individual tumor, margin analysis, and also immunohistochemistry (IHC) for therapy-relevant markers such as ER, PR, and HER2.

With regard to core needle biopsy or vacuum-assisted biopsy of mammographically suspicious lesions, excellent data are provided to use B-categorization for the description of needle biopsy specimens ( $\mathrm{LoE} 1 \mathrm{bB}, \mathrm{AGO}++$ ) [20, 173]. It is recommended to communicate the histopathological result within $24 \mathrm{~h}$ (at least dignity; LoE 5D, AGO +). We highly recommend immunohistological analysis of ER, PR and HER2 also in the needle biopsy specimens (3bC, AGO ++) [112,116, 178]. Fine-needle aspiration cytology does not represent state of the art for diagnosis of lumps or Breast Imaging Report and Data System (BI-RADS) IV/V lesions (LoE 5D, AGO -) $[107,196]$. However, although sensitivity is low, cytological examination of aspirated liquid from breast cysts or specimens of nipple secretion may be helpful in many cases (LoE $5 \mathrm{D}, \mathrm{AGO}+)$. Concerning handling and reporting of core needle biopsies, it is strongly recommended to participate in a quality assurance program ( $\mathrm{LoE} 3 \mathrm{D}, \mathrm{AGO}++)[5,37,108$, 114].

Sentinel lymph node excision may be an indication for immediate pathological analysis including frozen sections. However, it seems to be worthwhile only if it is followed by a 
clinical consequence ( $\mathrm{LoE} 5, \mathrm{AGO}+$ ). If no further axillary dissection is planned (according to the criteria of the ACOSOG Z0011-trial; see section 'Breast Cancer Surgery and Oncological Aspects') frozen sections of the excised sentinel node can be omitted (LoE 5D, AGO +/-) [206]. Today, tumor banking for research including clinical studies is getting more important. However, it is highly recommended to perform preservation of tumor tissue always in cooperation with the pathologist (LOE 5D, AGO +).

Pathological reporting must include tumor type and size, grade, tumor/node/metastasis (TNM) stage, margins in detail, extent of intraductal component if present, vascular invasion, and number of axillary lymph nodes involved, as well as ER, PR and HER2 expression.

Currently, Ki-67 is not recommended as a routine prognostic factor, but it may be helpful in validating the grade of tumors (LoE 3bB, AGO +). G1 tumors usually show a Ki-67 index below $15 \%$ and $\mathrm{G} 3$ tumors exhibit a labeling index $\geq 25 \%$. In core biopsies, $\mathrm{Ki}-67$ is better suited to predict the final histological grade than mitotic counts. Whether a threshold of $14 \%$ is able to discriminate between luminal A and B type awaits further research. The analysis of the 'International Ki-67 in Breast Cancer Working Group', however, has shown that $\mathrm{Ki}-67$ is highly sensitive to variations in methodology; therefore, it still cannot be recommended as standard marker [48, 57, 110, 126, 139, 203].

Special pathological reporting is necessary in patients undergoing neoadjuvant systemic therapy (NST). There exist at least 5 different reporting scores for the grade of remission after NST; some of these, however, are only validated for inflammatory breast cancer (e.g. [215]). If pathological complete remission ( $\mathrm{pCR}$ ) is expected, it seems to be prognostically relevant to report exactly the type of remission in detail, including information about remaining DCIS in the ipsilateral breast and remaining tumor in the ipsilateral axillary lymph nodes [198].

Determination of ER and PR has still to be performed by IHC. Results should be reported by percentage of stained tumor nuclei and tumors are called ER positive if $\geq 1 \%$ of the tumor nuclei are stained (LoE $1 \mathrm{aA}, \mathrm{AGO}++$ ). The staining intensity of the positive tumor nuclei may provide additional information. With regard to HER2 determination, only IHC and in situ hybridization (ISH) techniques (e.g. fluorescence ISH (FISH), chromogenic ISH (CISH)) are accepted (LoE $1 \mathrm{aA}, \mathrm{AGO}++)$. Results of dual-color ISH are judged positive if the signal ratio is $\geq 2.0$ (HER2 vs. centromer 17). Quality assurance evaluation showed that HER 2 overexpression is expected in $16 \%$ of unselected cases of EBC [37]. However, for ER/PR and HER2 expression, quantitative RNA assessment (qt-PCR, array) is not validated to estimate therapy sensitivity and must not be used (LoE 2bB, AGO -) [49, 132, 141].

From expression arrays, intrinsic types of breast cancer were derived: HER2-enriched, basal-like types, luminal type A and B. Although these molecular biologically defined types are highly suggestive for therapeutic decisions, it must not be disregarded that there is no generally accepted and proven translation into immunohistochemical counterparts, neither with regard to markers nor to thresholds [35, 36, 139, 152, 171].

In terms of practical consequences, re-labeling of clinically established and immunohistochemically defined subgroups may be useful (ER/PR+ for luminal type, HER2+ for HER2enriched type, triple-negative for basal type). The basal type shows an $80 \%$ overlap with the triple-negative subgroup of ductal invasive breast cancer $(\mathrm{ER}<1 \%$ and $\mathrm{PR}<1 \%$ and HER2 $0 / 1+2+$ non-amplified, ratio $<2.0)$. None of the available markers (Ki-67, grading, recurrence score, etc.) can reliably discriminate between luminal A and luminal B type [35].

Although derived from RNA expression studies, RNA measurements are not suited for the definition of intrinsic types for purposes of therapy [49].

With regard to the Genomic Health Recurrence Score (Oncotype $\mathrm{DX}^{\circledR}$ ), recently published data suggest that it may be substituted by a sophisticated algorithm based on immunohistochemical results (IHC4) [48].

\section{Prognostic and Predictive Factors}

In the past years, the use of biomarkers and molecular tests has been rapidly increasing in order to provide the patients with the optimal personalized treatment. On the other hand, many of the tests are not yet sufficiently validated [56].

The nuclear antigen Ki-67 has become established as a proliferation marker, although there has been a broad discussion on thresholds and lack of standardization. The AGO considers Ki-67 as an additional marker to further estimate the prognosis of EBC (LoE 1bB, AGO +) [179, 210]. In nodalnegative $\mathrm{EBC}$, proliferation markers like Ki-67 may be helpful in decision making ( $\mathrm{LoE} 2 \mathrm{bC}, \mathrm{AGO}+/-$ ) [179]. In the adjuvant setting in hormone receptor (HR)-positive patients receiving endocrine therapy alone, low Ki-67 was associated with good prognosis [48]. Furthermore, in adjuvant endocrine treatment, high Ki-67 levels ( $\geq 14 \%$ ) may identify patients that particularly benefit from initial letrozole/AI treatment versus tamoxifen (LoE 2aB, AGO +/-) [197]. On the other hand, Ki-67 in the daily practice might serve as predictive factor in favor of chemotherapy in the adjuvant setting [179] $(\mathrm{LoE} 2 \mathrm{aB}, \mathrm{AGO}+)$. Ki-67 may further predict response to neoadjuvant treatment [68] (refer to section 'Neoadjuvant [Primary] Systemic Therapy').

Topoisomerase II alpha (Topo IIa) gene amplification is a biomarker of potential usefulness in the neoadjuvant treatment of breast cancer. In endocrine-unresponsive and HER2-overexpressing tumors, Topo IIa amplification correlates significantly with a high probability of pCR after neoadjuvant anthracycline-based chemotherapy [156] (LoE 2bB, $\mathrm{AGO}+/-$ ). 
In nodal-negative patients with EBC, gene expression analysis may support decision making in some patients ( $\mathrm{LoE} 2 \mathrm{~b} \mathrm{~B}, \mathrm{AGO}+/-$ ). In view of the widespread use of those tests, the AGO committee provides a background chart in which 6 frequently used molecular predictors (Mammaprint, Oncotype DX, Theros, MapQuant Dx, Endopredict, and PAM 50) are screened. The distinct indications of the different tests have to be taken into account $[69,17,172,147,148]$. The molecular predictors should not be used to determine the HR status or especially the HER2 status [49].

\section{Breast Cancer Surgery and Oncological Aspects}

Clinical assessment combined with imaging is mandatory for preoperative assessment of the breast tumor. Preoperative histopathological diagnosis should be performed by imagingguided percutaneous needle biopsy (e.g. core needle biopsy) $(\mathrm{LoE} 1 \mathrm{cA}, \mathrm{AGO}+)$. The value of preoperative magnetic resonance imaging (MRI) to assess the extent of breast disease is still under discussion $(\mathrm{LoE} 1 \mathrm{cB}, \mathrm{AGO}+/-)$. This procedure may result in the detection of multiple invasive foci within the breast that had not been identified on previous standard mammography and ultrasonography. The impact of these findings on subsequent surgical management and ultimately disease-free survival (DSF) remains a controversial topic.

The standard surgical procedure for EBC is BCS followed by RT ( LoE 1aA, AGO ++). Skin-sparing mastectomy (SSM) with or without conservation of the nipple-areola complex is an oncologically safe treatment option [77, 146]. Survival rates of all these modern surgical procedures are at least equivalent to those after modified radical mastectomy (MRM).

Sentinel lymph node dissection (SLND) has become the standard surgical procedure in patients with clinically and sonographically negative axilla (cN0). SLND allows us to reliably stage patients as node positive or node negative [50, $76,160,184]$. Micrometastases $(\leq 2 \mathrm{~mm})$ in the sentinel lymph node are no indication for axillary dissection in clinically node-negative patients [72] $\left(\mathrm{LoE} 1 \mathrm{~b}^{\mathrm{a}} \mathrm{B}, \mathrm{AGO}-\right)$. The recent results from all SLND trials reflect that axillary dissection of lymph nodes may not not be necessary in patients with breastconserving therapy (BCT) if less than 3 sentinel lymph nodes are involved and the criteria of the ACOSOG study are met (LOE 2bB, AGO +/-). (ACOSOG criteria: The American College of Surgeons Oncology Group Z0011 trial, a phase III noninferiority trial conducted at 115 sites and enrolling patients from May 1999 to December 2004. Patients were women with BCT and clinical T1-T2 invasive breast cancer, no palpable adenopathy, and 1-2 sentinel lymph nodes containing metastases identified by frozen section, touch preparation, or hematoxylin/eosin staining on permanent sections [33, 207]). However, this is still an issue of debate since prospective studies with sufficient power are lacking [84]. If after thorough consideration surgeons and patients decide to omit axillary dissection, then irradiation of axillary lymph nodes is not indicated (LOE 5D, AGO -); whole-breast irradiation (WBI) with standard tangential fields is sufficient for local and regional control. However, axillary dissection remains mandatory in all cases with one or more involved sentinel lymph nodes if the inclusion criteria of the ACOSOG study are not met (e.g. mastectomy).

Surgery is an integral part of the neoadjuvant approach in breast cancer treatment. The aim of surgery after NST is to completely remove invasive and non-invasive breast cancer residues and to obtain clear margins at pathology examination. Important is the exact documentation of the tumor location before, during and after NST (LoE 5D AGO ++). Sentinel node excision should be performed prior to surgical procedures in order to obtain an exact staging ( $\mathrm{LoE} 3 \mathrm{bC} \mathrm{AGO} \mathrm{+)}$.

After primary surgery, adjuvant treatment (systemic therapy and RT) has to be started as soon as possible (LoE 1bA, AGO ++). Adjuvant chemotherapy should precede RT $(\mathrm{LoE} 1 \mathrm{bA}, \mathrm{AGO}++)$.

\section{Plastic Reconstructive Aspects after Mastectomy}

Oncoplastic surgery and breast reconstruction play an integral role in surgical treatment of breast cancer. In many women, postmastectomy breast reconstruction is essential to restore the body image and improve the quality of life. Timing of breast reconstruction is still a controversial issue [7, 83, 153, 177, 213]. Both options, immediate (IBR) and delayed breast reconstruction (DBR), are recommended on the same level $(\mathrm{LoE} 3 \mathrm{bB}, \mathrm{AG}++)$. Thus, the pros and cons have to be weighted against each other in each patient.

The main advantage of the DBR is the lack of interference with adjuvant procedures as chemotherapy and RT; a disadvantage is the loss of the skin envelope.

IBR is preferred in combination with BCS and in SSM. A recent Cochrane review [58] suggests, that IBR has the same complication rate as the delayed procedure, albeit based on very limited data (only 1 randomized controlled study with 64 women was found between 2008 and 2010). There is a need for prospective and longitudinal outcome studies analyzing this question.

If the necessity of postsurgical RT (PMRT) is not known at the time of mastectomy and reconstruction, there is the possibility of a delayed-immediate reconstruction. After SSM, an expander is implanted until the final decision about RT has been made. The planned standard reconstruction is performed after completion of RT or - if not necessary at all immediately ( $\mathrm{LoE} 3 \mathrm{bB}, \mathrm{AGO}+/-$ ).

In case of an SSM with IBR using silicone breast implants, the partially subpectoral pocket can be enlarged and secured by using mesh or an acellular dermal matrix [195]. However, 2 meta-analyses have shown that immediate postmastectomy implant reconstruction with acellular matrix had a higher 
complication rate than an expander implant, although with limited data for comparison $[2,105]$ ( $\mathrm{LoE} 2 \mathrm{~b} / 3 \mathrm{C}, \mathrm{AGO}+/-)$.

The issue of antibiotic prophylaxis remains controversial. In patients with postmastectomy implant reconstruction, especially after prior ipsilateral chest wall irradiation, a perioperative prolonged antibiosis (extended trimethoprim/sulfamethoxazole therapy) may be beneficial $(\mathrm{AGO}+)$. This was shown in a single-institution and single-surgeon analysis with 51 patients undergoing implant reconstruction from 2005 to 2008 [129].

\section{Adjuvant Endocrine Therapy in Premenopausal Patients}

The 2011 AGO recommendations for adjuvant endocrine therapy in premenopausal patients are still valid in 2012. The recent publication of the Early Breast Cancer Trialists' Collaborative Group (EBCTCG) meta-analysis strengthened the evidence of a substantial reduction of recurrence and mortality rates by 5 -year tamoxifen treatment of premenopausal ER-positive patients throughout the 15 years following randomization [61]. The ER status was the only predictive factor [61]. AIs alone must not be given in the premenopausal situation (LoE 1cA, AGO -). First results of ongoing studies (SOFT, TEXT) on the combination of gonadotropin-releasing hormone $(\mathrm{GnRH})$ agonists with tamoxifen or AIs are expected this year.

In some instances, the assessment of the ovarian reserve may be important in younger premenopausal as well as in perimenopausal patients. In perimenopausal women undergoing treatment for breast cancer, it may be difficult to determine the true menopausal status because adjuvant chemotherapy, tamoxifen, and GnRH analogs (GnRHa) can induce transient (or permanent) ovarian suppression. Measurement of anti-Muellerian hormone (AMH) and follicle count may add to the accuracy of hormone measures for determining the ovarian reserve (for both tests: $\mathrm{LoE} 3 \mathrm{bB}$; $\mathrm{AGO}+/-$ ).

(CRA) [175].

\section{Fertility Preservation}

Preservation of fertility is a priority in many younger women with EBC. Pretreatment AMH may be used to predict the long-term ovarian function after chemotherapy for EBC [6]. The antral follicle count appears to provide further knowledge of ovarian function, independent of $\mathrm{AMH}$ and other factors, e.g. follicle-stimulating hormone (FSH) or estradiol (E2) [176]. The antral follicle count is defined as the sum of follicle diameters of all follicles of $\geq 10 \mathrm{~mm}$ diameter in both ovaries [176].

Fertility preservation counseling is suggested in all patients who want to preserve their fertility ( $\mathrm{LoE} 4 \mathrm{C}, \mathrm{AGO}+)$. Assisted reproduction technology (ART) is an option for breast cancer survivors ( $\mathrm{LoE} 4 \mathrm{C}, \mathrm{AGO}+$ ).
The AGO recommendations concerning ovarian protection and fertility preservation in premenopausal patients receiving adjuvant chemotherapy did not change substantially compared to 2011. Whereas 4 observational studies reported an ovarian protective effect of GnRH agonists, only 1 of 3 recently published randomized controlled trials (RCTs) revealed a preventive effect. The ZORO study by Gerber et al. [78] concluded that the observed effect of GnRH agonists started prior to the 1st course of chemotherapy is, at its best, small. The study by Munster et al. [133] has not yet finished recruitment.

\section{Adjuvant Endocrine Therapy in Postmenopausal Patients}

Many updates of the well-known trials of AIs in adjuvant therapy have been published in 2011, confirming the current AGO recommendations.

Endocrine therapy is the most important systemic adjuvant therapy option in EBC. The EBCTCG meta-analysis in 2011, comprising data on the patient level of more than 20,000 patients over 15 years of observation time since randomization, consistently demonstrated a strong benefit with regard to recurrence rates und mortality for patients who received 5 years of adjuvant tamoxifen [61]. In this meta-analysis, it was further shown that endocrine therapy is only effective in ERpositive tumors but not in ER-negative tumors [59, 61, 101]. However, it was independent of adjuvant chemotherapy application.

This data results in the following therapy recommendations: Patients with receptor-negative tumors (ER- and PR-) should not receive endocrine therapy (LoE 1aA, AGO ++). Patients with receptor-positive tumors (ER+ and/or $\mathrm{PR}+$ ) should receive endocrine therapy $(\operatorname{LoE} 1 \mathrm{aA}, \mathrm{AGO}++)$. If chemo-endocrine therapy is indicated, endocrine treatment should be given sequentially after adjuvant chemotherapy rather than with chemotherapy (LoE 2bB, AGO ++).

With the current guidelines for receptor testing, all tumors with $1 \%$ or more of positive cells for ER and/or PR should be considered as hormone receptor positive, and the patients are therefore candidates for endocrine therapy [95].

The AGO Breast Committee introduced the following statement: Extended endocrine treatment (EAT) should be considered also for those patients who switched to the postmenopausal state during 5 years of tamoxifen treatment.

\section{Adjuvant Cytotoxic and Targeted Therapy}

Recently published trials and meta-analyses actually confirmed the AGO treatment recommendations with regard to adjuvant cytotoxic and targeted therapy. There were upgrades in the LoE and 2 clinically relevant modifications, one 
concerning trastuzumab in node-negative disease and the other focusing on other targeted agents (lapatinib, pertuzumab, and bevacizumab). In the following, these topics are described in the context of the main strategies in adjuvant therapy of EBC.

\section{Cytotoxic Agents and Combinations}

Provided that the indication for adjuvant chemotherapy is given, anthracycline-based combination chemotherapy is regarded as minimum standard treatment ( $\mathrm{LoE} 1 \mathrm{aA}, \mathrm{AGO}++)$. As shown in a meta-analysis, these regimens provide an additional reduction of relapse rate (ratio $0.89, \mathrm{p}=0.0001$ ) and mortality (ratio 0.84, $\mathrm{p}<0.001$ ) compared with adjuvant $\mathrm{CMF}$ therapy (cyclophosphamide, methotrexate, and 5-fluorouracil) after 15 years [59].

In general, by adding taxanes, DSF and overall survival can be further improved ( $\mathrm{LoE} 1 \mathrm{aA}, \mathrm{AGO}++)$ and eventually side effects, particularly cardiotoxicity, can be reduced. The corresponding relative risk reduction is rather dependent on the tumor biology than on the nodal status and extent of disease [62].

In node-positive disease, dose-dense ( $\mathrm{q} 2 \mathrm{w})$ adjuvant chemotherapeutic schedules may improve the relapse-free and overall survival compared to conventional adjuvant treatment options [22, 40, 130] (LoE 1aA, AGO ++). High-dose chemotherapy with autologous stem cell support is not recommended as adjuvant treatment in breast cancer ( $\mathrm{LoE} 1 \mathrm{aA}$, AGO -) [19].

The efficacy of CMF versus no chemotherapy has been shown in numerous trials with a long-term follow-up of meanwhile up to 20 years, and has been proven in meta-analyses (e.g. [62], EBCTCG 2012). Therefore, 6 cycles of CMF can be given in patients with contraindications for anthracycline- and taxane-containing regimens [21]. Still, in patients with a risk profile indicating a need for adjuvant chemotherapy, giving CMF is better than giving no therapy ( $\mathrm{LoE} 1 \mathrm{aA}, \mathrm{AGO}++$ ).

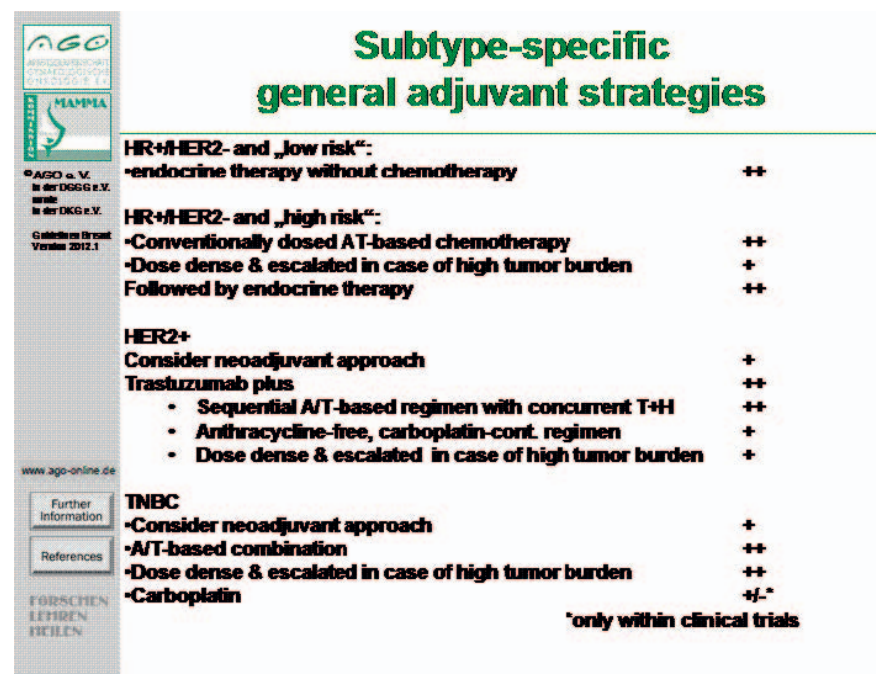

Fig. 1. Subtype-specific general adjuvant strategies.
Capecitabine, gemcitabine, and platinum compounds have been investigated in adjuvant trials. So far, none of these drugs can be recommended to be included into anthracycline/ taxane-based regimens. With regard to capecitabine, several trials could not demonstrate an additional benefit when adding capecitabine to an anthracycline/taxane-based therapy [e.g. 103, 144] (LoE 1bA, AGO +/-). Most of the trials used lower doses of either the taxane or capecitabine in order to cope with toxicity.

\section{Targeted Therapy}

Considering targeted therapy, all studies demonstrating a benefit for adjuvant trastuzumab in women with HER2positive tumors included node-negative and node-positive patients, and subgroup analysis showed a benefit for both groups of patients. Therefore, trastuzumab-containing regimens can be used in node-positive ( $\mathrm{LoE} 1 \mathrm{aA}, \mathrm{AGO}++$ ) as well as in node-negative patients, whenever chemotherapy is considered adequate. Women with node-negative disease, additional risk factors, and tumor size $>10 \mathrm{~mm}$ will profit from trastuzumab ( $\mathrm{LoE} 1 \mathrm{aA}, \mathrm{AGO}++)$. Moreover limited data from adjuvant studies suggest that even patients with HER2-positive tumors smaller than $10 \mathrm{~mm}$ may gain benefit from trastuzumab [87]. Thus, the use of trastuzumab may also be considered in smaller tumors (for tumors $>5-10 \mathrm{~mm}$, AGO +; for tumors $\leq 5 \mathrm{~mm}, \mathrm{AGO}+/-$ ).

In contrast to primary systemic therapy (PST), the phase III trials in adjuvant therapy with other targeted agents/dual HER2 blockade such as lapatinib, pertuzumab, and bevacizumab are not yet mature (recommendation level for all 3 compounds: $\mathrm{LoE} 5 \mathrm{D}, \mathrm{AGO}-$ ). In the TEACH trial, lapatinib was given in delayed adjuvant treatment, but the improvement of DSF (primary objective) did not reach statistical significance in [88] ( $\mathrm{LoE} 1 \mathrm{bB}, \mathrm{AGO}-)$.

\section{Subtype-Specific Strategies}

Molecular typing is based on molecular genetic testing and is not yet routinely available. Molecular subtypes and immunohistochemical surrogate markers of the tumors (HR, HER2, triple negative) correlate in about $70 \%$. The differentiation of luminal $\mathrm{A}$ and $\mathrm{B}$ subtypes by $\mathrm{HR}$ status and proliferation markers like Ki-67 may be helpful, but a prospective validation and standardization is still lacking. As prospective evidence is still pending, decision-making on chemotherapy based on genomic testing is not recommended outside clinical trials (see the following section). The current AGO expert opinion on treatment strategies in subtypes identified by IHC in a routine clinical setting is summarized in figure 1.

\section{Neoadjuvant (Primary) Systemic Therapy}

Recently published studies confirmed the benefit of NST and further specified its indications [51, 104, 189]. Retrospective 
analyses of subgroups defined by clinico-pathological parameters added to the knowledge of prognostic and predictive factors. In particular, recommendations emerged regarding modifications of cytotoxic regimens in cases of early or no early response to NST.

The achievement of pCR following NST is associated with improved survival [80, 109, 186, 198, 199]. Retrospective analyses suggested that this association is mainly confined to specific subgroups, in particular to triple-negative, HER2positive (non-luminal) and luminal B (HER2-negative) breast cancer $[66,199]$.

According to a meta-analysis that included 3332 patients treated in 7 German neoadjuvant trials, clinico-pathological factors predicting pCR following NST are younger age, smaller tumor size, non-lobular histology, higher grade, negative HR status, triple-negative, and HER2-positive status [43, 100, 200]. Considering subgroups, higher probability of pCR was associated with (1) longer treatment duration in HR-positive tumors, (2) higher doses of anthracyclines in HER2-negative tumors, (3) short-term higher doses of taxanes and anthracyclines in triple-negative tumors, and (4) trastuzumab-containing treatments in HER2-positive tumors [200].

Though results of exclusively retrospective studies evaluating gene expression signatures as prognostic and predictive parameters are promising, so far none of these signatures has been proven to be of sufficient discriminatory power to be used in the clinical setting [70] ( $\mathrm{LoE} 2 \mathrm{bC}, \mathrm{AGO}+/-$ ).

Outside clinical trials, the same regimens that are recommended in the adjuvant setting should be used for NST, i.e. anthracycline- and taxane-containing regimens for 18-24 weeks [104] (LoE 1aA, AGO ++). If adjuvant chemotherapy is indicated, the identical treatment can be applied in the neoadjuvant setting with similar DFS and overall survival, even over a follow-up period of 16 years [158]. Recommended regimens are those that were used in the superior treatment arms of large randomized neoadjuvant trials (NSABP B-27, GeparDuo, GeparTrio, ECTO).

A short dose-dense chemotherapy regimen with epirubicin and paclitaxel increased the pCR rate and survival as compared to 4 cycles of standard dose epirubicin plus paclitaxel [187]. A sufficiently long NST with dose-intensified epirubicin and paclitaxel followed by CMF, however, increased only the pCR rate but not the DSF as compared to standard treatment $[185,190]$ (LoE 1bB, AGO +/-).

Trastuzumab should be provided to all patients with HER2-overexpressing breast cancer and no contraindications $(\mathrm{LoE} 1 \mathrm{bA}, \mathrm{AGO}++)$ [29, 80, 81, 154, 188]. The dual antiHER2 treatment strategy with trastuzumab and lapatinib $(\mathrm{AGO}+/-)$ or trastuzumab and pertuzumab $(\mathrm{AGO}+/-)$ in combination with chemotherapy can significantly increase the pCR rate compared to a single anti-HER2 regimen. Survival data, however, are not available so far [12, 82, 91, 164]. Chemotherapy-free regimens combining 2 anti-HER2 agents were also active in a small subgroup of HER2-positive breast cancers. However, criteria to select patients for this approach are lacking [34, 82] (LoE 2bB, AGO +/-).

In patients with HER2-negative breast cancer, 2 large randomized phase III studies showed a higher pCR rate with neoadjuvant chemotherapy plus bevacizumab than with chemotherapy alone, but survival data are still lacking and subgroup analyses showed inconsistent results [15, 75, 201]. In the German GeparQuinto trial, bevacizumab significantly increased the $\mathrm{pCR}$ rate in the triple-negative subgroup, while no effect was seen in HR-positive patients [75, 201]. In the NSABP B40 trial, however, the effect of bevacizumab was seen predominantly in HR-positive breast cancer patients [15] (for both subgroups: AGO +/-).

Tumor response to NST is evaluated by clinical examination or ultrasound or mammography. In case of early response following 2-4 courses (6-12 weeks) of an anthracyclinecontaining NST, the AGO Breast Committee recommends to complete NST as planned for at least 18 weeks in accordance with Kaufmann et al. [104] (LoE 1bA, AGO ++). In patients with HR-positive tumors responding to 2 cycles of docetaxel, adriamycin, cyclophosphamide (DAC), however, continuation of treatment with 6 instead of 4 cycles of DAC significantly improved DFS and overall survival. In a retrospective subgroup analysis, this benefit was confined to patients with HR-positive breast cancer [199] (LoE 2b ${ }^{\mathrm{a}} \mathrm{C}$, $\mathrm{AGO}+)$.

In patients with no early response to NST (no change), completion of chemotherapy as planned is recommmended $(\mathrm{LOE} 2 \mathrm{bC}, \mathrm{AGO}++)$. An alternative option is continuation of NST with a non-cross-resistant regimen, as is 4 cycles of paclitaxel or docetaxel following 4 cycles of adriamycin-cyclophosphamide ( $\mathrm{LOE} 2 \mathrm{bB}, \mathrm{AGO}+$ ). In case of no change to 2 cycles of DAC, the switch to 4 cycles of vinorelbine plus capecitabine also improved survival (LOE 1bB, AGO ++).

In case of progressive disease, stop of PST and immediate surgery or RT is recommended (LOE 4D, AGO ++). Additional adjuvant chemotherapy with a non-cross-resistant regimen may be considered (LOE 4D, AGO +/-).

Another important objective of NST is reduction in tumor volume, thus making BCT feasible. BCT should not be considered if negative margins are not achievable even after repetitive excisions [104] ( $\mathrm{LoE} 3 \mathrm{bC}, \mathrm{AGO}-$ ). BCT is further not indicated in case of widespread DCIS or microcalcifications (AGO -) and should not be considered if adjuvant RT is not feasible [104] (AGO -).

In inflammatory breast cancer, BCS is not recommended, even if clinically complete response has been achieved (LoE 2bC, AGO -); in case of pCR, however, BCT may be considered although data are scarce $(\mathrm{AGO}+/-)$. Selected patients with multicentric lesions (lesions in different quadrants) or cT4a-c tumors who achieved a clinically complete response with NST had good outcome following BCT in small retrospective analyses. However, BCT is not recommended in 
these cases as a routine procedure outside controlled clinical trials, but may be an option in individual cases (AGO +/-) $[51,104]$.

\section{Adjuvant Radiotherapy}

\section{EBCTCG Update 2011}

Postoperative RT is known to substantially reduce the risk of local and regional breast cancer relapse, both when given after mastectomy (MRM) and after BCS. The recently published update of the EBCTCG analysis clearly demonstrates an overall survival benefit due to adjuvant RT after BCS (15year gain $3.8 \%$ with RT for all patients, $3.4 \%$ in pN0 and $8.5 \%$ in $\mathrm{pN}+$ patients) [60]. Absolute decrease in the risk of relapse of $16 \%$ (19\% vs. $35 \%)$ resulted in an absolute decrease of $4 \%(21 \%$ vs. $25 \%)$ in the risk of dying from breast cancer [27]. RT led to a proportional reduction of $50 \%$ in overall recurrence (referring mainly to locoregional recurrences), thus exceeding that from chemotherapy or endocrine therapy alone [27]. In 2009, similar analyses were published from the Surveillance, Epidemiology and End Results (SEER) and the UZ Brussels databases [204].

Even in patients with invasive breast cancers and presumably favorable prognostic factors (tumor size $<1 \mathrm{~cm}$, ER positivity, no ipsilateral lymph node involvement, low grading), ipsilateral breast cancer recurrence after BCS occurs frequently enough to justify postoperative irradiation, regardless of adjuvant systemic endocrine treatment $[1,41,59,60,121$, 209].

In the EBCTCG update 2011 concerning adjuvant RT in breast cancer patients after BCS, the ' $4: 1$ relation' was confirmed: For every 4 recurrences avoided by year 10 , about 1 breast cancer death is avoided by year 15 [27, 60]. The same relation has been seen before in breast cancer patients with RT after mastectomy [27].

To obtain the advantages of adjuvant RT, the use of modern techniques in RT planning and delivery is mandatory.

\section{WBI and Hypofractionation}

WBI should be systematically applied after BCS (LoE 1aA, AGO ++). The total dose should be equal or equivalent to $50 \mathrm{~Gy}$ in 25 fractions over 5 weeks. RT of the breast in addition to BCS reduces the risk of relapse in all patient subgroups and is strongly recommended in the updated guidelines of the National Comprehensive Cancer Network (NCCN) USA (2012), the National Institute for Health and Clinical Excellence (NICE) CG80 (2009), the Belgian Health Care Knowledge Centre (KCE) (2010), the New Zealand Guidelines Group (2009), and the French Expert Review Board (2011) [16, 32, 135, 136, 138].

Although the issue of fractionation of RT of the whole breast is still subject of discussion in international societies of radiation oncology, regarding hypofractionated WBI, new data from RCTs are convincing and gave reason for a change in recommendations [96, 209]. In the update 2012, the AGO Breast Committee states that hypofractionation schedules in WBI should be considered in selected patients ( $\mathrm{LoE} 1 \mathrm{aB}$, $\mathrm{AGO}+$ ).

The recommendation is in part based on new evidence resulting from a systematic review of the Cochrane Collaboration [102]. Reviewing the data of RCTs dealing with hypofractionated RT, the authors substantially altered their conclusion of the last review from 2008, stating: 'Two new studies have been published since the last version of the review, altering our conclusions. ... We have evidence from four low to medium quality randomized trials that using unconventional fractionation regimens (greater than 2 Gy per fraction) does not affect local recurrence, is associated with decreased acute toxicity and does not seem to affect breast appearance or late toxicity for selected women treated with BCT. These are mostly women with node negative tumors smaller than $3 \mathrm{~cm}$ and negative pathological margins' [102].

Four trials, involving 7095 women, were included in the review. Local recurrence was not significantly different for women having fewer treatments. Most of the women in the trials $(89.8 \%)$ had tumors less than $3 \mathrm{~cm}$ in size, all had complete removal of the tumor on pathology and $79 \%$ had no evidence of cancer in their lymph nodes. Where the breast size was known, $87 \%$ had small or medium breasts. This updated Cochrane Collaboration review indicates that for women who fit these criteria, using fewer radiation treatments after tumor removal is a safe and effective option [102].

Additionally, hypofractionated RT schemes are further recommended/considered in several updated international guidelines (Belgian KCE 2010, French guidelines 2010, NCCN 2011, NICE 2010).

Nevertheless, caution is still warranted because long-term follow-up ( $>5$ years) is available for only a small proportion of the randomized patients (Canadian study [207]).

Longer follow-up is required for a more complete assessment of the effect of altered fractionation [102].

\section{Accelerated Partial Breast Irradiation}

The AGO Breast Committee did not change the recommendations concerning accelerated partial breast irradiation (APBI) as a prior ('anticipated') or postoperative boost RT $(\mathrm{LoE} 1 \mathrm{aA}, \mathrm{AGO}+)$ [183]. Growing evidence suggests that APBI alone may be able to sufficiently reduce the risk of local relapses in selected patients. On the other hand, APBI as sole therapy still remains an experimental approach due to the lack of results from long-term follow-up of RCTs (LoE 1bC, AGO -). Thus, partial breast irradiation should be performed as part of prospective trials. Partial breast irradiation can be delivered using interstitial, intracavitary, externalbeam or intraoperative techniques (IORT) (for details see www.ago-online.de). 


\section{Radiotherapy of the Axilla}

The AGO recommendations regarding RT of the axilla have been modified in view of the corresponding recommendations for axilla surgery. If in case of positive sentinel lymph nodes axillary lymph node dissection (ALND) is not performed in compliance with the recommendations stated in the section 'Breast Cancer Surgery and Oncological Aspects', WBI with standard tangential fields might be sufficient for local and regional control; additional separate $\mathrm{RT}$ of the axilla may be harmful and is not indicated [194] (LoE 2aB, AGO -).

\section{Internal Mammary Chain Radiotherapy}

Important prospective data suggested that internal mammary chain RT would not be necessary, even in cases of internal or central tumor locations, or in patients with positive axillary lymph nodes. These data warrant confirmation. The indications for internal mammary chain RT should be carefully balanced against potential toxicity ( $\mathrm{LoE} 2 \mathrm{bC}, \mathrm{AGO}+/-$ ). High-quality techniques should be used for decreasing the dose delivered to the heart [8].

\section{Specific Situations in Breast Cancer}

\section{Older Patients}

Increasing evidence suggests that axilla dissection can be omitted in case of small tumors and clinically negative axilla $(\mathrm{LoE} 2 \mathrm{bC}, \mathrm{AGO}+)$. Recently, Martelli et al. [122] presented the update of a study including 671 patients $\geq 70$ years (172 with axilla dissection and 499 patients without). After a median follow up of 15 years there was no significant difference in mortality rates in patients with pT1 $\mathrm{cN} 0$ disease with and without axilla dissection, respectively (10.7\% vs. $10.7 \%$, $\mathrm{p}=0.836)$.

\section{Inflammatory Breast Cancer}

Prospective randomized studies in patients with inflammatory breast cancer are still missing. Current updates are focusing on the definition that demands definitive diagnosis of invasive carcinoma and clinical signs of skin affection $(>1 / 3$ of the breast involved, in contrast to the previous definition of $>2 / 3$ involvement) [51]. Biopsies of the skin should be obtained for diagnostic reasons ( $\mathrm{LoE} 2 \mathrm{cB}, \mathrm{AGO}+$ ), yet only with a detection rate of $<75 \%$. The use of trastuzumab as neoadjuvant treatment option for inflammatory breast cancer (LoE 2bB, $\mathrm{AGO}++$ ) is further supported by the current data of the NOAH study [166]. In inflammatory breast cancer, BCS is not recommended, even if a clinically complete response is achieved by neoadjuvant chemotherapy ( $\mathrm{LoE} 2 \mathrm{bC}, \mathrm{AGO}-$ ); in case of pCR, however, BCS may be considered, although data are scarce $(\mathrm{AGO}+/-)$. [51, 104].

\section{Carcinoma of Unkown Primary}

RT of the ipsilateral breast may be considered if axillary metastases are detected in patients suffering from carcinoma of unkown primary (CUP) with inconspicious MRI of the breast (LoE 3bC, AGO +/-). 48 patients with negative MRI results were included into a non-randomized study; herein, $73 \%$ were treated with radiation and $27 \%$ were observed. After a median follow-up of 68 months, recurrence-free survival was shown in $84 \%$ patients with RT versus $34 \%$ without $(\mathrm{p}<0.001)[11]$.

\section{Phyllodes Tumor and Paget's Disease}

Recommendations for malign phyllodes tumor as well as Paget's disease did not undergo any changes due to lack of new data and clinical trials.

\section{Sarcoma (Angiosarcoma) of the Breast}

Current data show that in sarcoma, particularly angiosarcoma, not the type of surgery but the size of the tumor, grading, and especially adequate safety margins are decisive prognostic factors ( $\mathrm{LoE} 4 \mathrm{C}, \mathrm{AGO}++$ ). Angiosarcoma is a serious disease that can occur 10-15 years after RT. BCS may be performed under the condition that large safety margins are feasible and the patient has given her informed consent in view of the associated risk (LoE 4C, AGO +/-) [4, 205]. Diagnosis should be obtained by core biopsy (LoE 4D, AGO ++), not fineneedle biopsy (LoE 4D, AGO -) [119]. After surgery, anthracycline-based chemotherapy and RT might be considered, particularly in high-risk cases ( $\mathrm{LoE} 4 \mathrm{C}, \mathrm{AGO}+/-$ ) [10]. In metastatic disease, paclitaxel as well as liposomal doxorubicin should be given, particularly in patients with angiosarcoma. After treatment failure with anthracycline and ifosfamide, trabectedin can be offered to patients suffering from leiomyosarcoma (LoE 2bB, AGO +) [165].

\section{New Aspects in Therapy Side Effects}

\section{Bisphosphonates and Denosumab}

In terms of toxicity, bisphosphonates and denosumab seem to be similar (e.g. [174]). In patients with advanced breast cancer receiving bisphosphonates and denosumab, the rates of adverse events (AEs) and serious AEs were similar. Osteonecrosis of the jaw (ONJ) occurred infrequently (2.0\% denosumab; $1.4 \%$ zoledronic acid; $\mathrm{p}=0.39$ ); however, patients should be informed about this specific risk [174]. In a recently published pooled analysis of 3 randomized phase III trials of denosumab versus zoledronic acid in patients treated for $\mathrm{MBC}$, the occurrence rate of ONJ for denosumab was confirmed with $1.67 \%$ [191]. 


\section{Bevacizumab}

Recently published systematic reviews are corresponding with toxicity data of randomized trials with bevacizumab plus chemotherapy. In an open-label, randomized, phase III trial, the efficacy and safety of paclitaxel plus bevacizumab was compared with that of paclitaxel alone in patients with MBC. Severe AEs were more frequent in patients who received paclitaxel plus bevacizumab than in the paclitaxel alone group: grade 3 or 4 hypertension $(14.8 \%$ vs. $0.0 \%, \mathrm{p}<0.001)$, proteinuria $(3.6 \%$ vs. $0.0 \%, \mathrm{p}<0.001)$, headache $(2.2 \%$ vs. $0.0 \%$, $\mathrm{p}=0.008)$, and cerebrovascular ischemia $(1.9 \%$ vs. $0.0 \%$, $\mathrm{p}=0.02)$. Infection was more common $(9.3 \%$ vs. $2.9 \%$, $\mathrm{p}<0.001)$ in patients receiving the combination, but febrile neutropenia was uncommon $(<1 \%$ overall) [128].

A systematic review and meta-analysis of 5 randomized phase III clinical trials that used bevacizumab alone or in combination with chemotherapy in MBC showed a statistically significant bevacizumab-associated increased risk for proteinuria (odds ratio $(\mathrm{OR})=27.68)$, hypertension $(\mathrm{OR}$ $=12.76)$, left ventricular dysfunction $(\mathrm{OR}=2.25)$, and hemorrhagic events $(\mathrm{OR}=4.07)$, while no increased incidence was found for gastrointestinal perforation, vascular or fatal events and febrile neutropenia [44] (for a review of side effects see also [94]). However, careful selection of patients for this therapy seems to be reasonable.

\section{Bisphosphonates and RANKL Antibody Denosumab}

Bone health and thus osteooncology is gaining increasing importance in the management of breast cancer patients. New data have been published in 2011 for bisphosphonates and denosumab resulting in changes in current AGO recommendations [183].

In adjuvant treatment, bisphosphonates can be recommended with a high level of evidence for the treatment and prevention of cancer-related loss in bone mineral density (BMD) (LoE 1bB, AGO ++). BMD should be measured regularly (LoE 2bB, AGO +). Besides physical activity, avoiding a body mass index $(\mathrm{BMI})<20 \mathrm{~kg} / \mathrm{m}^{2}$, sufficient calcium intake, and vitamin $\mathrm{D}$ supplementation, the reduction of smoking is recommended ( $\mathrm{LoE} 4 \mathrm{C}, \mathrm{AGO}++$ ).

The RANK ligand (RANKL) antibody denosumab can be considered as promising alternative to bisphosphonates in the treatment of osteoporosis (LoE 1bA, AGO ++) [47]. Denosumab, however, is not yet licensed for the prevention of bone loss in breast cancer patients ( $\mathrm{LoE} 1 \mathrm{bB}, \mathrm{AGO}+$ ), although data is available. The effect of denosumab on BMD was evaluated in women receiving adjuvant AIs. After 24 months, the lumbar spine BMD increased by $7.6 \%$, the hip BMD by $4.7 \%$, and the distal radius by $6.1 \%[63,64]$. Ongoing studies currently evaluate this effect in the adjuvant setting.

With regard to prevention of metastases, bisphosphonates (clodronate $1600 \mathrm{mg}$ /day p.o. or zoledronic acid $4 \mathrm{mg}$, q6m, i.v.) are indicated in specifically defined subgroups of patients $(\mathrm{LoE} 1 \mathrm{bB}, \mathrm{AGO}+)[42,52,86,150]$. In an update of the ABCSG-12-trial, zoledronic acid significantly improved DFS (hazard ratio (HR) 0.71 ; 95\% confidence interval (CI) 0.55 $0.92 ; \mathrm{p}=0.011)$ [86].

In postmenopausal women on adjuvant treatment with zoledronic acid and letrozole, the 5-year results of the ZOFAST study revealed a continuous increase of the BMD and an improvement of the DFS by $34 \%$ [52]. Furthermore in a subgroup analysis of the AZURE study, the DFS increased in postmenopausal women (HR 0.75; 95\% CI 0.59-0.96; p = 0.02 ) as well as the overall survival (HR 0.74; $95 \%$ CI $0.55-$ $0.98 ; \mathrm{p}=0.04$ ) [42]. Bisphosphonates, however, are not yet licensed for the prevention of metastases.

To date, bisphosphonates are the current standard in the therapy of patients suffering from breast cancer-related bone metastases (LoE $1 \mathrm{aA}, \mathrm{AGO}++)$. In the registration trial, Stopeck et al. [174] demonstrated a significant advantage of denosumab (120 mg s.c., q28d) compared to zoledronic acid ( $4 \mathrm{mg}$ i.v., q28d) regarding the time to occurrence of a skeletal-related event (SRE) (HR 0.82; $\mathrm{p}=0.0096)$. The time period until the occurrence of moderate and severe pain could be prolonged from 176 to 295 days ( $p=0.0024)$.

The results were confirmed through integrated analyses of 3 denosumab pivotal trials [115]. The time period to the reoccurrence of events was prolonged. Additionally, the type of SRE, for example the reduction of pathological fractures or the necessity of RT, showed consistent results [85]. Thus, the application of denosumab is a promising alternative for the reduction of SRE ( $\mathrm{LoE} 1 \mathrm{aA}, \mathrm{AGO}++)$ as well as for the prolongation of the time period to the occurrence of bone pain $(\mathrm{LoE} 1 \mathrm{bA}, \mathrm{AGO}++)$.

Before starting the treatment with bisphosphonates or denosumab, the women should be informed on possible unwanted side effects. Furthermore, due to the risk of ONJ in the range of $1.3-1.8 \%$ (LoE $1 \mathrm{~b}$ ), the patients should be offered a complete dental examination and, if necessary, oral restorative and rehabilitation therapy (LoE $1 b)$.

\section{Supportive Care}

There have been no clinically relevant changes in the AGO recommendations compared to 2011. Managing breast cancer with highly effective cytotoxic therapies, it is essential to recognize febrile neutropenia, which is defined by the following criteria: oral temperature $>38.5{ }^{\circ} \mathrm{C}$ or 2 consecutive readings $>38{ }^{\circ} \mathrm{C}$ within $2 \mathrm{~h}$ in a patient with an absolute neutrophil count $(\mathrm{ANC})<500$ cells $/ \mu$ l or expected to fall below 500 cells/ $\mu$ l. Thorough baseline clinical examination and daily clinical evaluation is mandatory (LoE 5D AGO ++). Alhough in general patients should be hospitalized (LoE 1bA $\mathrm{AGO}++)$, some low-risk patients may be treated also by homecare (LoE 1bA AGO +). Routinely, differential blood 
cell count, blood cultures, and lung imaging have to be performed. Antibiotic therapy has to be initiated immediately based on empirical efficacy (LoE 1aA AGO ++) - and adjusted later. In case of failure of antibiotic therapy, empirical antifungal therapy must also be initiated for 4-7 days (LoE 1bA AGO ++). Application of granulocyte colonystimulating factor $(\mathrm{G}-\mathrm{CSF})$ is a matter of debate (LoE $2 \mathrm{~b} B$ $\mathrm{AGO}+/-)$.

\section{Breast Cancer Follow-Up}

While there were no clinically relevant modifications in the existing AGO recommendations for breast cancer follow-up, in 2012 new statements were included concerning LCIS and DCIS. Women with LCIS and DCIS have an elevated risk for breast cancer. Thus risk communication should provide women with information of risk reduction strategies (e.g. follow-up schedule and medical intervention). Follow-up of women with LCIS or DCIS comprises interval history, physical examinations and counseling every 6-12 months for 5 years, and then annually. A diagnostic mammography should be performed yearly. In patients undergoing BCT for invasive breast cancer, the first ipsilateral follow-up mammogram should be performed 6-12 months after the completion of breast-conserving RT) [136].

\section{Locoregional Recurrence}

The AGO recommendations for locoregional recurrence did not change substantially in 2012 as compared to 2011. Some trials and systematic reviews that were published in 2011 add to the knowledge about risk factors.

About $10 \%(2-20 \%)$ of patients who underwent BCT will subsequently develop ipsilateral breast tumor recurrence (IBTR). Chest wall recurrences after mastectomy and isolated axillary recurrences are relatively rare events. Risk factors for IBTR include tumor size, nodal status, ER status, young age, positive microscopic margins, higher grading, vessel invasion, an extensive intraductal component, and lymphatic vessel invasion. Systemic treatment and RT significantly reduced local recurrence. HER2 positivity emerged as an independent risk factor in addition to ER and PR negativity, basal-like and triple-negative tumors [30, 118, 192].

Although the local outcome following salvage therapy is quite good, the risk of distant metastases for patients with local recurrence is 3-5 times higher than for those without recurrence. The patients with locoregional recurrence survived almost significantly better than those with distant recurrence. The interval between primary diagnostics and recurrence correlated positively with overall survival after a recurrence [113]. The risk of distant metastases/impaired survival was increased if $\geq 2$ factors of the following were positive: lymphovascular space invasion (LVSI), higher grading, ER negativity, and close margins [149].

Isolated IBTRs following BCT have a better prognosis with regard to overall survival probability (5-year overall survival: $65 \%$ ) than chest wall recurrences following mastectomy (5-year overall survival: $50 \%$ ). If the supraclavicular fossa is additionally affected, the overall survival probability decreases (3-year overall survival: 49\%) [159].

The analyses also showed that patients with early recurrence had an impaired survival probability whilst in patients with late recurrences survival probability is not affected [113].

There are no studies that confirm an implication of the re-staging findings in systemic treatment or improvement of overall survival of asymptomatic patients with resectable locoregional recurrence. Nevertheless, to avoid 'over- or undertreatment' and to prevent complications, the AGO recommends re-staging in all patients with locoregional recurrences as in 2011 ( $\mathrm{LoE} 5 \mathrm{D}, \mathrm{AGO}++$ ).

\section{Endocrine Therapy in MBC}

Hormonal therapy remains the treatment of first choice for metastatic patients with endocrine-responsive breast cancer ( $\mathrm{LoE} 1 \mathrm{a} \mathrm{A}, \mathrm{AGO}++)$. The sequential use of the available drugs depends on the individual pretreatment. Reassessment of ER, PR, and HER2 in tumor tissue at the time of diagnosis of relapse is recommended whenever possible, in order to prove metastatic disease and to better tailor treatment. In a prospective analysis, therapeutic management for 1 of 6 patients was changed after reassessment of metastases [182]. However, it has not yet been investigated whether the efficacy of treatment is superior if it would be guided by the new ER, PR or HER2 status of the current metastasis rather than if it is based only on the findings of the primary tumor.

Fulvestrant plays an increasing role in the treatment of MBC. In addition to the approved dose of $250 \mathrm{mg}$ by intramuscular injection, a high-dose (HD) regimen of fulvestrant (500 mg once a month plus $500 \mathrm{mg}$ on day 14 of month 1 ) is associated with a significantly longer progression-free survival (PFS) and can be recommended for patients who had progressed on prior endocrine therapy (LoE 1bA, AGO +). Based on currently available data, HD fulvestrant appears to be as well tolerated as standard fulvestrant. However, no survival benefit was shown $[46,98]$.

In 707 patients with HR-positive disease, first-line endocrine treatment with anastrozole was compared to the combination of anastrozole and fulvestrant. Fulvestrant doses were 500, 250 and $250 \mathrm{mg}$ at start and after 14 and 28 days, respectively. Thereafter, fulvestrant was given with $250 \mathrm{mg}$ every 4 weeks. For the whole study population, PFS (13.5 vs. 15.0 months; HR 0.80; 95\% CI 0.68-0.94; $\mathrm{p}=0.007)$ and overall survival (41.3 vs. 47.7 months; HR 0.81; 95\% CI 0.65-1.00; 
$\mathrm{p}=0.049)$ were statistically superior in favor of the combination therapy versus anastrozole alone. Unplanned analysis by prior tamoxifen suggested benefit only in the tamoxifen-naive group [125].

Everolimus and exemestane was compared to exemestane and placebo (randomly assigned in a 2:1 ratio) in 724 postmenopausal women with HR-positive advanced breast cancer who progressed while receiving previous therapy with a nonsteroidal AI in the adjuvant setting and/or advanced disease. Overall response $(0.4$ vs. $9.5 \%$; $<<0.001)$ and clinical benefit rate were significantly higher in the combination group versus exemestane alone. Further, the median PFS was significantly increased with the combination both by local (2.8 vs. 6.9 months; HR 0.43 ; $95 \%$ CI $0.35-0.54$; $\mathrm{p}<0.001$ ) and central assessment (4.1 vs. 10.6 months; HR 0.36; 95\% CI $0.27-0.47 ; \mathrm{p}<0.001)$. Data on survival is immature. The combination therapy was associated with a higher incidence of AEs than with exemestane alone. The AEs observed with everolimus plus exemestane included stomatitis, fatigue, pneumonitis, diarrhea, pyrexia, and hyperglycemia [13]. The data are convincing, such that the combination exemestane/ everolimus may be used in some patients as second-line endocrine treatment (LoE 1bA, AGO +). No changes and modifications of the current recommendations compared to 2011 have been made for premenopausal, HR-positive, HER2-negative women and for postmenopausal, HR-positive, HER2positive patients.

\section{Cytotoxic Therapy in MBC}

In palliative therapy after anthracycline treatment, taxanes are the most commonly used compounds. A Cochrane analysis demonstrated a modest survival advantage of taxane-based versus non-taxane regimens in this situation [79].

Nanoparticle albumin-bound paclitaxel (nab-paclitaxel) has demonstrated significant activity in MBC. A phase III trial showed higher response rates and time to progression of nab-paclitaxel compared with paclitaxel $\left(175 \mathrm{mg} / \mathrm{m}^{2}\right.$ over $3 \mathrm{~h}$ every 21 days) [90]. Nab-paclitaxel was associated with a lower risk of hypersensitivity reactions, less grade 4 neutropenia, but increased grade 3 sensory neuropathy. Recently, a phase II randomized study showed longer PFS of nab-paclitaxel compared with docetaxel [89]. The AGO recommendation for its use in second-line treatment was strengthened $(\mathrm{LoE} 2 \mathrm{bB}, \mathrm{AGO}++)$. However, neither docetaxel nor nabpaclitaxel has demonstrated superiority over the weekly schedule of paclitaxel [89].

There are few effective treatment options available to women with MBC who failed to respond or relapsed after receiving both anthracyclines and taxanes. Several agents, including capecitabine, vinorelbine, gemcitabine, epothilones, and platinum salts, as well as combinations of these, have been investigated [145]. Re-challenge with taxanes [93] or eribulin may also be beneficial after failure of anthracyclines and taxanes.

In a recent open-label phase III trial $(n=762)$ [45], eribulin mesylate, a non-taxane microtubule inhibitor, was compared to treatment of physician's choice (TPC). Overall survival was significantly improved in women assigned to eribulin (median 13.1 months compared with TPC (10.6 months; $\mathrm{p}=0.041)$ $(\mathrm{LoE} 1 \mathrm{bB}, \mathrm{AGO}++)$. The most common AEs in the eribulin group were asthenia or fatigue, neutropenia, and peripheral neuropathy.

Ixabepilone is active in anthracycline- and taxane-pretreated patients [131]. A recent phase III study demonstrated that the addition of ixabepilone to capecitabine is associated with increased response rates and PFS compared with capecitabine alone [181]. Yet, this benefit was achieved at the expense of increased toxicity, particularly sensory neuropathy and neutropenia. Moreover, ixabepilone is not registered for MBC in Germany (LoE 1bB, AGO -).

\section{Specific Situations and Sites of Metastases}

New data from recent studies and systematic reviews in 2011 substantially confirmed the AGO recommendations.

In metachronous or primary $\mathrm{MBC}$, management focuses on systemic therapy. The impact of local treatment on overall survival is still under discussion. With regard to surgery or RT for the primary tumor, however, retrospective data on more than 30,000 women from North America and Europe have now been published, showing a robust association between those procedures and prolonged survival [151]. The question remains whether this association reflects a selection of women with good prognosis [157]. There are also reports that could not find an advantage in overall survival after local surgery in this situation [55]. The AGO considers mastectomy or local excision of the primary tumor as an option for selected patients (AGO $+/-$ ). Axilla surgery is only indicated in patients with bulky axillary disease.

In distant metastases, surgery should be in general considered in patients with a good health condition, oligometastases, and a long distance between primary treatment and the occurrence of metastases [140, 163]. Reported improved overall survival might be the result of patient selection. Before surgery is done, metastases should be confirmed by histology [23] $(\mathrm{LoE} 3 \mathrm{~B}, \mathrm{AGO}+)$. After surgery, local and systemic treatment according to guidelines should be considered. Other indications for surgical intervention are symptoms like persisting pain or exulceration after systemic treatment (LoE 5D, $\mathrm{AGO}+/-)$.

Resection of liver metastases should only be performed if $\mathrm{R} 0$ resection is feasible and no extrahepatic metastases are present $[18,38,99,120]$ (LoE 3bC, AGO +/-). Other procedures like regional $\mathrm{RT}$ as well as thermoablation are indicated in individual cases $(\mathrm{AGO}+/-)$. Mostly a survival benefit for 
surgery or other ablation techniques has been reported. However, this might be the result of patient selection.

For proven pulmonary metastases, the level of evidence for a curative approach is low, but some patients might benefit from metastasectomy followed by an appropriate systemic treatment (e.g. [111]) ( $\mathrm{LoE} \mathrm{3bC,} \mathrm{AGO} \mathrm{+/-).} \mathrm{In} \mathrm{accordance}$ with the treatment of liver metastases, resection of lung metastases should only be performed if $\mathrm{R} 0$ resection is feasible and if histological verification was done. Other procedures like thermoablation are indicated in individual cases (LoE 3bC, $\mathrm{AGO}+/-)$.

\section{Bone Metastases}

There were no clinically relevant changes compared to 2011 except for new data and new recommendations with regard to the treatment with bisphosphonates and denosumab (see section 'Bisphosphonates and RANKL Antibody Denosumab').

\section{Central Nervous System Metastases in Breast Cancer}

For many years it has been debated whether patients with a limited number of brain metastases (BM) may benefit from a whole-brain RT (WBRT) after local treatment of these lesions. Recently published data suggest that such an additional procedure should be restricted to individual cases because overall survival was not prolonged [106]. Moreover, preliminary results of a multicenter phase II trial indicate that an upfront therapy with lapatinib and capecitabine could be an option for patients with multiple BM caused by HER2-positive breast cancer [9].

\section{Targeted Therapy in MBC}

Despite many recently published and further ongoing studies examining targeted therapy in breast cancer, recommendations in the update 2012 with regard to MBC are confined to 2 major clinical relevant topics: the combination treatment with pertuzumab plus trastuzumab plus docetaxel in HER2positive $\mathrm{MBC}$ and the combination everolimus plus exemestane in HR-positive MBC; both have got the recommendation level $\mathrm{AGO}+$. Another field of clinically relevant discussion focuses on the evaluation of bevacizumab combination treatment with diverse cytotoxic agents. (For targeted therapy combined with cytotoxic agents in the adjuvant setting, see the respective section.)

\section{Pertuzumab}

Pertuzumab, an anti-HER2 humanized monoclonal antibody that inhibits receptor dimerization, has a mechanism of action that is complementary to that of trastuzumab. In the recently published phase III study, 808 patients with HER2-positive MBC received pertuzumab plus trastuzumab plus docetaxel or trastuzumab, docetaxel alone as first-line treatment. Addition of pertuzumab significantly improved the primary end point PFS [14]. Median PFS was 18.5 months in the pertuzumab group versus 12.4 months in the control group (HR for progression or death $0.62 ; 95 \%$ CI $0.51-0.75$; $\mathrm{p}<0.001$ ). Interim analysis of overall survival showed a trend in favor of pertuzumab. There was no increase in left ventricular systolic dysfunction; the rates of febrile neutropenia and diarrhea grade $\geq 3$ were higher in the pertuzumab group. This new combination expands the choice of possible treatment options based on trastuzumab ( $\mathrm{LoE} 1 \mathrm{bA}, \mathrm{AGO}+)$. Since pertuzumab is still not approved, study participation is strongly recommended.

\section{Everolimus plus Exemestane}

Data of a recently published randomized phase III trial showed that everolimus combined with exemestane improved PFS in patients with HR-positive advanced breast cancer previously treated with nonsteroidal AIs compared to exemestane alone [13].

Resistance to endocrine therapy in breast cancer is associated with activation of the mammalian target of rapamycin (mTOR) intracellular signaling pathway. The mTOR inhibitor everolimus added to endocrine therapy may enhance antitumor activity. Included in the trial were 724 patients with HR-positive advanced breast cancer who had recurrence or progression while receiving previous therapy with a nonsteroidal AI in the adjuvant setting or in advanced disease. Median PFS (primary endpoint) was 10.6 months with everolimus and exemestane versus 4.1 months in the control group (HR 0.36; 95\% CI 0.27-0.47; $\mathrm{p}<0.001$ ). The AGO level of recommendation is $\mathrm{LoE} 1 \mathrm{bB}, \mathrm{AGO}+$; yet, study participation is recommended.

\section{Bevacizumab Combination Treatment}

Another treatment option in MBC is to target the vascular endothelial growth factor (VEGF). The diverse regimens were re-evaluated by the AGO experts. In first-line treatment in HER2-negative MBC, the combination of bevacizumab and paclitaxel was equally well recommended (LoE $1 \mathrm{bB}$, $\mathrm{AGO}+$ ) as the regimen bevacizumab plus capecitabine (LoE 2bB, $\mathrm{AGO}+)[25,128,162,169]$.

\section{Complementary Therapy, Hormonal Treatment, and Alternatives for Breast Cancer Survivors}

This year's update sees quite a few substantial modifications as compared to the 2011 version. They were brought about by a number of relevant publications, and particularly the publication of several important Cochrane reviews.

It has now been shown that prophylactic lymph drainage as an adjunct to guideline-conform care including physical therapy (exercise) has no significant impact on the short-term development of arm lymph edema following breast and axillary surgery [54] (LoE 1a B, AGO -). 
Early exercise therapy after surgery, however, does help to improve upper limb dysfunction, but increases daily wound drainage loss and prolongs the need for drainage therapy [123] (LoE 1a A, AGO +). Additionally, the Cochrane review on the value of thymic peptides in cancer treatment has finally been published: There was preliminary evidence that thymic peptides lowered the risk of severe infectious complications in patients undergoing chemotherapy or RT, but there was no convincing indication that thymic peptides could help to im prove cancer survival [208] (LoE 2a B, AGO +/-). Finally, new evidence accumulates that phytoestrogens are more likely friend than foe in the fight against BC. Flaxseed, an im portant source of enterolactone-bearing lignans, has once more been investigated in breast cancer survivors. Buck et al. [28] found in 1140 postmenopausal breast cancer patients that high serum enterolactone levels were associated with impro ved survival. These findings were supported by a recent metaanalysis indicating a significant inverse association between serum enterolactone and postmenopausal breast cancer risk, which was stronger for ER- PR- than for ER+ PR+ tumors [211]. Interestingly, soy food consumption was significantly associated with decreased risk of death and recurrence in Chinese breast cancer survivors [167]. The AGO grade of recommendation for soy food for the whole group of patients with advanced breast cancer is LoE $2 \mathrm{~b}$ B, AGO +/-, and for the patients with HR-positive breast cancer LoE $2 b$, AGO -. In contrast to earlier reports, vitamin use shortly after breast cancer diagnosis was associated with reduced mortality and recurrence risk [137]. The AGO judgment for antioxidants after finishing RT is LoE $2 b B, A G O+/-$. How ever, the effects of antioxidant supplement use after diagnosis differ by the type of antioxidants administered.

While certain Chinese medicinal herbs seem to alleviate aspects of treatment-related side effects [212], the Cochrane review by Milazzo et al. [127] warns that laetrile lacks any promise of efficacy but could bear significant risks due to unforeseen toxicity (LoE 1c D, AGO -).

Breast cancer patients require comprehensive guidance with respect to possibilities, effectiveness, and side effects of complementary and alternative therapeutic approaches. Therefore, practicing physicians are required to inform them selves about the expectations of their patients. Not infre quently, these expectations with regard to the efficacy of complementary and alternative medicine (CAM) are unreal istically exaggerated. Only cooperative guidance of patients by informed oncologists can prevent communication break down and avert the danger of conflicting therapies and drug interferences.

\section{Options for Primary Prevention: Modifiable Lifestyle Factors}

There have been no substantial changes in the AGO recom mendations compared to 2011.

\section{Disclosure Statement}

The members of the editing committee of these guidelines are specialists in diagnosis, treatment, and research in breast cancer. Most of the members therefore cooperate with the industry. Thus, potential conflict of interest cannot be excluded. In order to minimize potential bias within the statements, we followed these predefined rules: These guidelines are strictly based on available evidence from the scientific literature; the chapters of each edition were prepared by annually alternating teams of authors; each statement and the correspondent AGO recommendations were thoroughly discussed within the entire group and accepted by majority decisions; each member of the editing committee is required to submit a written declaration of his/her conflicts of interest to an elected internal Conflicts of Interest (COI) committee on an annual basis; members who do not submit a COI declaration may not participate in the guideline preparation. All members of the AGO Breast Committee have submitted their COI report for the past year. Members of the AGO Breast Committee indicated that they have received support (e.g. re search funding, lecture or consulting honoraria, etc.) from the following entities: Abraxis BioScience Ltd., Amgen GmbH, AstraZeneca GmbH, Bayer-Schering Pharma, Boehringer Ingelheim Pharma $\mathrm{GmbH}$ and $\mathrm{Co}$. KG, Cephalon GmbH, Chugai Pharma Marketing Ltd., GBG For schungs-GmbH, Genentech, Genomic Health, GlaxoSmithKline GmbH and Co. KG, Johnson and Johnson $\mathrm{GmbH}$, Medac mbH, MediGene AG, Mentor $\mathrm{GmbH}$, MSD Sharp and Dohme $\mathrm{GmbH}$, Mundipharma mbH and Co. KG, Novartis Pharma GmbH, Pfizer Pharma GmbH, Pierre Fabre Pharma GmbH, Roche Pharma AG, Sanofi-Aventis GmbH, Siemens AG. The Committee did not consider any of the reported sup port to represent a conflict of interest that would preclude participation in AGO Breast Committee discussions or voting.

\section{References}

To access the references please refer to www.karger.com/?DOI=000342279. 